\title{
An eye for discovery
}

\author{
Andreas Stahl and Lois E.H. Smith \\ Department of Ophthalmology, Children's Hospital, Harvard Medical School, Boston, Massachusetts, USA
}

\begin{abstract}
Vision research has often led to significant advances in our understanding of biology. There has also been particular success in translating basic research in the eye into breakthrough clinical therapies that mark important milestones for ophthalmology and also for medical research. Anti-VEGF therapy for age-related macular degeneration was named as one of the top ten science advancements of the year 2006. Only two years later, successful transfer of the RPE65 gene into retinal pigment epithelium of patients with Leber congenital amaurosis was noted as one of the most important clinical applications of gene therapy. The articles in this Review series outline current developments in vision research and highlight its continued importance in ophthalmology and medicine.
\end{abstract}

\section{Introduction}

The ancient proverb "the eye is the window to the soul" is echoed prosaically in science. Vision research has long led to a deeper knowledge of biology, as illustrated by the Nobel Prize-winning work by David Hubel and Torsten Wiesel over 30 years ago. They defined neural structure-function relationships of sensory information processing in the visual cortex. They also defined windows of cortical plasticity that for the first time explained how vision loss in amblyopia arises from visual sensory deprivation during a critical developmental period. Their work remarkably advanced our understanding of not only visual information processing but also of brain microarchitecture and function in general. Similarly important, work conducted by George Wald in the 1930s found visual pigments in the retina linking vitamin A to phototransduction, leading to his Nobel Prize in 1967.

Ophthalmology has also been at the forefront of translational research. Basic eye research (1-3) was translated to the clinic with anti-VEGF therapy for age-related macular degeneration (AMD). This was voted in 2006 by Science magazine as among the top ten science breakthroughs of the year (4) after two clinical trials $(5,6)$ showing that treatment with the anti-VEGF drug ranibizumab improves vision in one-third of patients with wet AMD while stabilizing the condition in most of the remaining patients. This is in stark contrast to all prior treatments for AMD, in which loss of fewer than three lines of visual acuity had been considered a success. Eye research trials have also played critical roles in advancing human gene therapy as a therapeutic principle for clinical practice. Transfer of the RPE65 gene into retinal pigment epithelium (RPE) of patients with Leber congenital amaurosis (LCA), a retinal degenerative disease that had previously been considered untreatable, can partially restore visual function $(7,8)$. The RPE65 gene therapy trials are some of the most successful applications of gene therapy in humans to date.

This Review series discusses a wide range of current vision research developments and highlights how vision research continues to advance the fields of both ophthalmology and medicine (Figure 1).

\section{Stemming vision loss}

Marchetti et al. discuss the potential use of stem cells for rebuilding both vascular and neuronal retinal tissue (9). Particularly intriguing is the possibility of using induced pluripotent stem cells (iPSCs) in the treatment of damaged RPE in AMD. AMD is the

Conflict of interest: The authors have declared that no conflict of interest exists. Citation for this article: J Clin Invest. 2010;120(9):3008-3011. doi:10.1172/JCI44158. most common neurodegenerative retinopathy and a major cause of blindness $(10,11)$. RPE cells, whose dysfunction may be one of the earliest events in the pathogenesis of $\operatorname{AMD}(12,13)$, form a single-layered interface between the photoreceptor layer of the retina and the underlying choroidal vessels and play a vital role in photoreceptor metabolism and survival. Loss or damage of RPE cells leads to secondary photoreceptor loss in the affected area. Stem cell-based techniques to replace or repair damaged RPE could therefore have great potential for halting AMD development at an early stage. Marchetti et al. discuss the advantages and limitations of using iPSCs in this context as well as for other neurodegenerative eye disorders with primary photoreceptor damage, such as retinitis pigmentosa. The review also offers an overview of ocular stem cell research in the anterior segment of the eye for reepithelialization of damaged cornea as well as trabecular meshwork-derived stem cells or neuronal stem cells for treatment of glaucoma. However, replacing ganglion cells lost to glaucoma is a daunting challenge, with the problem of "rewiring" newly grown neuronal cells to their appropriate target areas in the cortex. More success with stem cell therapy - at least in animal models - has already been achieved in the treatment of primary vascular diseases such as retinopathy of prematurity (ROP). Both endothelial cell progenitor cells (EPCs) and bone marrow-derived myeloid cells have successfully been used to prevent or reduce retinal vascular damage and the subsequent neuronal degeneration resulting from poor retinal perfusion $(14,15)$.

\section{Through the eyes of a child}

An in-depth analysis of the pathogenesis of ROP and proliferative retinopathy is given in the review by Sapieha and colleagues (16). The study of ROP has led to a greater understanding of angiogenesis, vascular neural interconnections, and metabolic vascular crosstalk. The authors illustrate in detail how in the premature infant, exposure of the developing retina to unphysiologically high oxygen tension leads first to arrest of normal vascular development (phase I of ROP) and subsequently to excessive and uncontrolled blood vessel growth (phase II). Based on the lack of autonomic vascular innervation of the retina (17-19), the retinal vasculature relies predominantly on local factors to regulate tissue oxygen tension. In the prematurely born infant, however, local autoregulation of both retinal and choroidal blood flow is very limited compared with that in adults (20). This dysregulation in combination with oxygen levels that by far exceed the in utero levels normally present at this stage of retinal development leads to abnormally high tissue oxygen tension. One direct consequence is the generation of ROS and reactive 


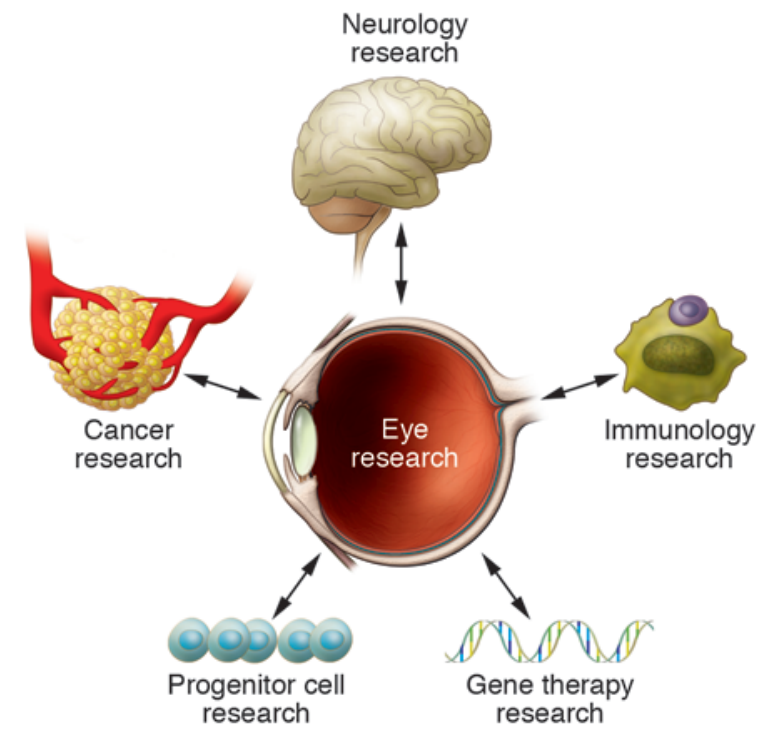

Figure 1

Eye research and its multiple connections to other areas of medical research.

nitrogen species (RNS), which contribute to retinal microvascular damage. Beyond these direct toxic effects, oxygen also suppresses oxygen-regulated growth and cell-survival factors of both vascular and neuronal cells. Low levels of VEGF during early ROP result in poor vascular development and areas of avascular retina. During the second phase of ROP, these avascular areas become hypoxic and VEGF and other angiogenic growth factors become massively upregulated. This excessive overproduction of hypoxia-induced vasoactive growth factors, however, leads to disorganized and misdirected growth of blood vessels from the retina into the normally avascular vitreous, causing sight-threatening complications such as tractional retinal detachment, bleeding, and retinal edema. The Review especially emphasizes the importance of neuronal and neuroglial cells during this second phase of ROP. Retinal ganglion cells (RGCs) and astrocytes of the inner retina in particular accumulate Krebs cycle intermediates from incomplete oxidative metabolism. Some of these intermediates, most notably succinate and adenosine, trigger an angiogenic response even before Hif $1 \alpha$ stabilization, the classic inducer of hypoxia-dependent gene transcription, is established (21). Recent studies in animal models of ROP have identified succinate and GPR91 as a novel ligand-receptor pair linking cellular metabolism to angiogenesis. GPR91 and succinate are likely to also play significant roles in the pathogenesis of other ocular and nonocular neoangiogenic processes such as, for example, cancer growth.

\section{Time's eye}

Focusing on a condition related to aging, the review by Alan Bird discusses the pathophysiology and current treatment options for AMD (22). This review summarizes our current understanding of local alterations at the posterior pole of the eye that are associated with the initiation and progression of, in particular, the "dry" (i.e., nonvasoproliferative) form of AMD. During the early stages of disease, focal deposits, termed drusen, are found in the Bruch membrane between RPE and choroidal vessels. While the neighboring RPE often exhibits irregular pigmentary changes, vision is usually normal or only mildly impaired at this early stage. With further progression, however, thickening of the Bruch membrane, focal reduction in choroidal capillary density, RPE cell loss, and photoreceptor degeneration can result in more significant visual impairment. Unfortunately from a functional point of view, all these changes (in their most complete form often referred to as geographic atrophy) appear almost exclusively in and around the macula, the part of the retina that is responsible for central vision and higher visual functions such as reading or recognizing faces. The Review highlights the structural changes seen specifically in AMD eyes, but also discusses the complex involvement of several systemic factors in detail. Although the author points out that the increased prevalence of AMD seen in population-based studies appears not to be solely attributable to an increased life expectancy (23), systemic aspects of aging can be unraveled in the study of AMD. Dysregulation of the immune system especially in the complement cascade and lifestyle-associated risk factors such as smoking and obesity are important systemic contributors to AMD progression. The identification of AMD-associated haplotypes in the gene for complement factor $\mathrm{H}$, in particular, has significantly advanced the field of AMD research and has contributed substantially to integrating this local degenerative disease of the eye into a wider picture of systemic immune function.

\section{Nudging nature}

As with AMD, recent advances in molecular genetics have helped to reveal the underlying defects in many inherited retinal dystrophies. Based on these diagnostic achievements, gene therapy has advanced to a successful clinical debut in treatment studies in patients with LCA due to defects in the RPE65 gene $(7,8)$. The Review by den Hollander et al. discusses the recent breakthroughs in diagnosing and treating retinal inherited disorders, a heterogenous group of blinding eye diseases that until recently were considered incurable (24). While affecting a variety of different genes, all retinal dystrophies discussed in this Review are characterized by the dysfunction and eventual degeneration of photoreceptor and/or RPE cells. The connection between underlying gene defect and clinical presentation is in many cases not easy to make, and the distinction among some retinal dystrophies can be very subtle or even arbitrary. Thus an essential prerequisite for any successful gene therapy is the exact identification of the underlying genetic defect in each patient. den Hollander and colleagues provide an overview of the current standards in molecular genetics and discuss the need for reliable animal models for preclinical testing of gene therapy approaches. A challenge going forward is how to adapt gene therapy strategies for RPE treatment to the treatment of photoreceptors, as these cells often carry the genetic deficit responsible for retinal dystrophies. This will involve adjusting the vector system and the injection protocols and could potentially be associated with risks such as ectopic vector expression in brain cells. While the success of the LCA-RPE65 trials may have depended on treating patients with useful vision early in life, the seminal results obtained from these first ocular gene therapy trials offer real hope for patients with other forms of retinal dystrophy and put ocular research at the forefront of human gene therapy development.

\section{Seeing is believing}

The Review by Blumberg and Kreiman provides an overview of visual information processing (25). They focus on object recognition, a crucial cerebral visual-processing function necessary for reading or face identification. The capacity of the human visual system to 
recognize faces is still much more powerful than computer-based systems in part because of the robustness of our visual system in accommodating image transformations. Even when most of the pixels of an image are changed or the object is altered in size, position, luminance, motion, or texture, the human brain still can recognize and name that object appropriately. Electrophysiological studies on animals as well as patients with spatially defined brain lesions have helped to map specific areas of the cortex involved in image recognition. In addition, neurophysiological studies with epileptic patients have provided evidence that the recognition of faces can be used to probe the robustness of image recognition despite image alteration. In one patient, a particular face no matter how transformed elicited a selective response in one particular neuron. A response specific to this face occurred whether it was presented as a black and white drawing, as a color photograph, or in different poses and views (26). These results help to advance our understanding not only of the connections between eyes and brain, but also our understanding of the complex interactions triggered by sensory input into the cerebral cortex, perhaps leading, among other achievements, to more advanced computer face recognition.

\section{Fittest for survival}

All of the Reviews discussed so far focus on either ocular or cerebral disorders of vision. The Review by Fan and Wiggs concentrates on RGCs that link the retina to the first interconnecting neurons of the cerebral visual pathways (27). RGCs are the cells primarily damaged in glaucoma, leading to severe visual field defects and, if left untreated, complete blindness. Current treatments can prevent progression of ganglion cell loss in the majority of glaucoma patients; they are, however, not curative. Many forms of glaucoma appear to be inherited. Most genes identified to date lie within 29 genetic loci $(28,29)$ and are mostly associated with ocular development. As a consequence, mutations in these genes most commonly lead to early onset or juvenile glaucoma. However, mutations in some genes, such as optineurin (30) and WDR36 (31), are also found in patients with the more prevalent adult-onset form of primary open angle glaucoma (POAG) and are thought to directly affect RGC survival.

Interesting questions regarding the interplay between systemic and ocular abnormalities are raised by studies showing an overlap of genetic regions involved in regulation of intraocular pressure and regulation of blood pressure (32). Given that genetic testing can identify individuals at risk for early onset glaucoma in approximately $30 \%$ of cases (33), gene therapy might potentially offer a targeted treatment option with long-lasting effect in at least certain subgroups of glaucoma patients similar to treatments for retinal dystrophies as discussed in the Review by den Hollander et al. (24). Finally, analogous to the repair of neuronal or vascular retinal tissue as discussed in the review by Marchetti et al. (9), the use of pluripotent stem cells for either supply of neurotrophic factors or facilitation of endogenous repair of damaged RGCs might become a suitable treatment option for glaucoma patients in the future.

\section{Eyeing immunity}

The Review by Rachel Caspi offers another link between vision research and advances in other areas of medical research (34). Her article on uveitis highlights the special relationship between the immune system and ocular tissues. While the eye enjoys a so called "ocular immune privilege" (35), uveitis is nevertheless often associated with systemic immunological disorders such as juvenile rheumatoid arthritis, ankylosing spondylitis, or Behçet disease. Common predisposing factors for both the ocular and the systemic manifestations of the underlying immunologic disorders are HLA haplotypes. Particularly striking is the association between Vogt-Koyanagi-Harada syndrome or systemic ophthalmia and HLA DR4 and birdshot retinochoroidopathy and HLA A-29. This association of uveitic diseases with certain HLA haplotypes has been explored in the commonly used mouse model of experimental autoimmune uveitis (EAU). The retinal antigen to which uveitis patients most frequently respond is arrestin (S-Ag). Arrestin is, however, a poor uveitogen in the EAU mouse model except in transgenic mice expressing HLA DR4 (36).

The Review also provides a thorough overview of the current therapies available to treat uveitis. One treatment strategy that has evolved from animal models into clinical practice is the targeting of pathogenic effector $\mathrm{T}$ cells using cyclosporine, FK-506, or rapamycin. Other approaches include administration of appropriate doses of retinal uveitogenic antigens to correct defects in peripheral antigen tolerance by infusion of autologous B cells engineered to express uveitogenic antigen fragments. Finally, treatment strategies targeting pathogenic effector cytokines such as TNF- $\alpha$ and IL-17 or blocking adhesion molecules and inflammatory recruitment factors have shown promising results. Notably, many of these treatments are not unique for uveitic disorders but show promising effects also in systemic conditions such as arthritis and thus further illustrate how ocular research is interconnected with a wide array of various medical research areas.

\section{Conclusion}

The Review series presented in this issue of JCI provides an overview of the wide and promising field of ophthalmology research. The universal importance of some of the findings presented highlights the role of the eye and the visual system as a research tool for other biomedical specialties. Irrespective of whether a particular researcher's interest lies in immunology, angiogenesis, gene therapy, or neurodevelopment, the eye is an easily accessible organ that can be used to visualize a wide variety of physiological and pathological concepts.

\section{Acknowledgments}

A. Stahl is supported by the Deutsche Forschungsgemeinschaft (DFG). L.E.H. Smith is supported by the NIH (EY017017, EY017017-04S1), the V. Kann Rasmussen Foundation, the Roche Foundation for Anemia Research, the Research to Prevent Blindness Senior Investigator Award, the Alcon Research Institute Award, the Lowy Medical Research Institute (MacTel), Children's Hospital Boston Mental Retardation and Developmental Disabilities Research Center.

Address correspondence to: Lois E.H. Smith, Children's Hospital, Department of Ophthalmology, 300 Longwood Ave., Boston, Massachusetts 02115, USA. Phone: 617.355.8531; Fax: 617.734.5731; E-mail: lois.smith@childrens.harvard.edu.

\footnotetext{
1. Adamis AP, et al. Inhibition of vascular endothelial growth factor prevents retinal ischemia-associated iris neovascularization in a nonhuman primate. Arch Ophthalmol. 1996;114(1):66-71.
}

2. Aiello LP, et al. Suppression of retinal neovascularization in vivo by inhibition of vascular endothelial growth factor (VEGF) using soluble VEGF-receptor chimeric proteins. Proc Natl Acad Sci U S A.
1995;92(23):10457-10461.

3. Robinson GS, Pierce EA, Rook SL, Foley E, Webb $\mathrm{R}$, Smith LE. Oligodeoxynucleotides inhibit retinal neovascularization in a murine model of 
proliferative retinopathy. Proc Natl Acad Sci U S A. 1996;93(10):4851-4856.

4. [No authors listed]. Breakthrough of the year. The Runners-Up. Science. 2006;314(5807):1850-1855.

5 . Brown DM, et al. Ranibizumab versus verteporfin for neovascular age-related macular degeneration. NEngl J Med. 2006;355(14):1432-1444.

6. Rosenfeld PJ, et al. Ranibizumab for neovascular age-related macular degeneration. $N$ Engl J Med. 2006;355(14):1419-1431.

7. Bainbridge JW, et al. Effect of gene therapy on visual function in Leber's congenital amaurosis. NEngl JMed. 2008;358(21):2231-2239.

8. Maguire AM, et al. Safety and efficacy of gene transfer for Leber's congenital amaurosis. $N E n g l$ J Med. 2008;358(21):2240-2248.

9. Marchetti V, Krohne TU, Friedlander DF, Friedlander M. Stemming vision loss with stem cells. J Clin Invest. 2010;120(9):3012-3021.

10. Congdon $\mathrm{N}$, et al. Causes and prevalence of visual impairment among adults in the United States. Arch Ophthalmol. 2004;122(4):477-485.

11. Resnikoff S, et al. Global data on visual impairment in the year 2002. Bull World Health Organ. 2004;82(11):844-851.

12. Zarbin MA. Current concepts in the pathogenesis of age-related macular degeneration. Arch Ophthalmol. 2004;122(4):598-614.

13. de Jong PT. Age-related macular degeneration. NEngl J Med. 2006;355(14):1474-1485.

14. Otani A, et al. Rescue of retinal degeneration by intravitreally injected adult bone marrow-derived lineage-negative hematopoietic stem cells. J Clin Invest. 2004;114(6):765-774.

15. Ritter MR, Banin E, Moreno SK, Aguilar E, Dorrell MI, Friedlander M. Myeloid progenitors differen- tiate into microglia and promote vascular repair in a model of ischemic retinopathy. J Clin Invest. 2006;116(12):3266-3276.

16. Sapieha P, et al. Retinopathy of prematurity: understanding ischemic retinal vasculopathies at an extreme of life. J Clin Invest. 2010; 120(9):3022-3032.

17. Ehinger B, Falck B. Concomitant adrenergic and parasympathetic fibres in the rat iris. Acta Physiol Scand. 1966;67(2):201-207.

18. Hoste AM, Boels PJ, Andries LJ, Brutsaert DL, De Laey JJ. Effects of beta-antagonists on contraction of bovine retinal microarteries in vitro. Invest Ophthalmol Vis Sci. 1990;31(7):1231-1237.

19. Laties AM. Central retinal artery innervation. Absence of adrenergic innervation to the intraocular branches. Arch Ophthalmol. 1967;77(3):405-409.

20. Milligan DW. Failure of autoregulation and intraventricular haemorrhage in preterm infants. Lancet. 1980;1(8174):896-898.

21. Sapieha P, et al. The succinate receptor GPR91 in neurons has a major role in retinal angiogenesis. Nat Med. 2008;14(10):1067-1076.

22. Bird AC. Therapeutic targets in age-related macular disease. J Clin Invest. 2010;120(9):3033-3041.

23. Evans J, Wormald R. Is the incidence of registrable age-related macular degeneration increasing? $\mathrm{Br} \mathrm{J}$ Ophthalmol. 1996;80(1):9-14.

24. den Hollander AI, Black A, Bennett J, Cremers FPM. Lighting a candle in the dark: advances in genetics and gene therapy of recessive retinal dystrophies. J Clin Invest. 2010;120(9):3042-3053.

25. Blumberg J, Kreiman G. How cortical neurons help us see: visual recognition in the human brain. J Clin Invest. 2010;120(9):3054-3063.

26. Quiroga RQ, Reddy L, Kreiman G, Koch C,
Fried I. Invariant visual representation by single neurons in the human brain. Nature. 2005;435(7045):1102-1107.

27. Fan BJ, Wiggs JL. Glaucoma: genes, phenotypes, and new directions for therapy. J Clin Invest. 2010;120(9):3064-3072.

28. Fan BJ, Wang DY, Lam DS, Pang CP. Gene mapping for primary open angle glaucoma. Clin Biochem. 2006;39(3):249-258.

29. Wiggs JL. Genetic etiologies of glaucoma. Arch Ophthalmol. 2007;125(1):30-37.

30. Rezaie T, et al. Adult-onset primary open-angle glaucoma caused by mutations in optineurin. Science. 2002;295(5557):1077-1079.

31. Hauser MA, et al. Distribution of WDR36 DNA sequence variants in patients with primary open-angle glaucoma. Invest Ophthalmol Vis Sci. 2006;47(6):2542-2546

32. Duggal P, Klein AP, Lee KE, Klein R, Klein BE, Bailey-Wilson JE. Identification of novel genetic loci for intraocular pressure: a genomewide scan of the Beaver Dam Eye Study. Arch Ophthalmol. 2007;125(1):74-79.

33. Mackey DA, Craig JE. Predictive DNA testing for glaucoma: reality in 2003. Ophthalmol Clin North Am. 2003;16(4):639-645.

34. Caspi RR. A look at autoimmunity and inflammation in the eye. J Clin Invest. 2010;120(9):3073-3083.

35. Medawar PB. Immunity to homologous grafted skin; the fate of skin homografts transplanted to the brain, to subcutaneous tissue, and to the anterior chamber of the eye. Br J Exp Pathol. 1948;29(1):58-69.

36. Pennesi G, et al. A humanized model of experimental autoimmune uveitis in HLA class II transgenic mice. J Clin Invest. 2003;111(8):1171-1180. 